\title{
TEMPLATED GROWTH OF METAL-ORGANIC COORDINATION CHAINS AT SURFACES
}

$>$ Th. Classen, G. Costantini, F. L. Stadler, C. Kim, K. Kern

Max-Planck-Institut fuer Festkoerperforschung, Heisenbergstr. 1, 70569 Stuttgart, GERMANY

$>$ G. Fratesi, S. Fabris, S. de Gironcoli, S. Baroni

SISSA and INFM-CNR DEMOCRITOS National Simulation Center, Via Beirut 2-4, 34014 Trieste, ITALY

Metal-organic coordination networks (MO$\mathrm{CNs}$ ) formed by coordination bonding between metallic centers and organic ligands can be efficiently engineered to exhibit specific magnetic, electronic, or catalytic properties [1]. Instead of depositing prefabricated MOCNs onto surfaces, it has been recently shown that two-dimensional (2D) MOCNs can be directly grown at metal surfaces under ultrahigh vacuum (UHV), thus creating highly regular 2D networks of metal atoms [2]. We show here [3] that this approach allows to predefine the geometry of the MOCN by using the substrate as a template to direct the formation of novel $1 \mathrm{D}$ metal-organic coordination chains (MOCCs).

The templating role of substrates is well known in the field of surface epitaxial growth. Among the highly anisotropic substrates, the $\mathrm{Cu}(110)$ surface is one of the most commonly used. To demonstrate its strong 1D templating effect on organic molecules, a ligand with a triangular symmetry was selected, namely 1,3,5-benzenetri-car- boxylic acid (trimesic acid, TMA). The three-fold rotation symmetry of TMA supports the formation of hexagonal 2D and 3D architectures, therefore strongly disfavoring the linear geometry.

The deposition of TMA on $\mathrm{Cu}(110)$ under UHV at $300 \mathrm{~K}$ results in the formation of $1 \mathrm{D}$ chains along the $<1$ bar $10>$ direction, as observed by scanning tunneling microscopy (STM). This deposition temperature is high enough to provide mobile $\mathrm{Cu}$ adatoms through evaporation from kinks and steps onto the terraces. Analysis of similar systems by X-ray photoelectron spectroscopy showed that these adatoms catalyze the deprotonation of molecular carboxylate groups and are necessary for the formation of copper carboxylate complexes. The chains formed at $300 \mathrm{~K}$ typically show irregular kinks and poor long-range order. These inhomogeneities are removed by postannealing to $380-410 \mathrm{~K}$ to yield straight and highly periodic chains, referred to as MOCC-I hereafter.
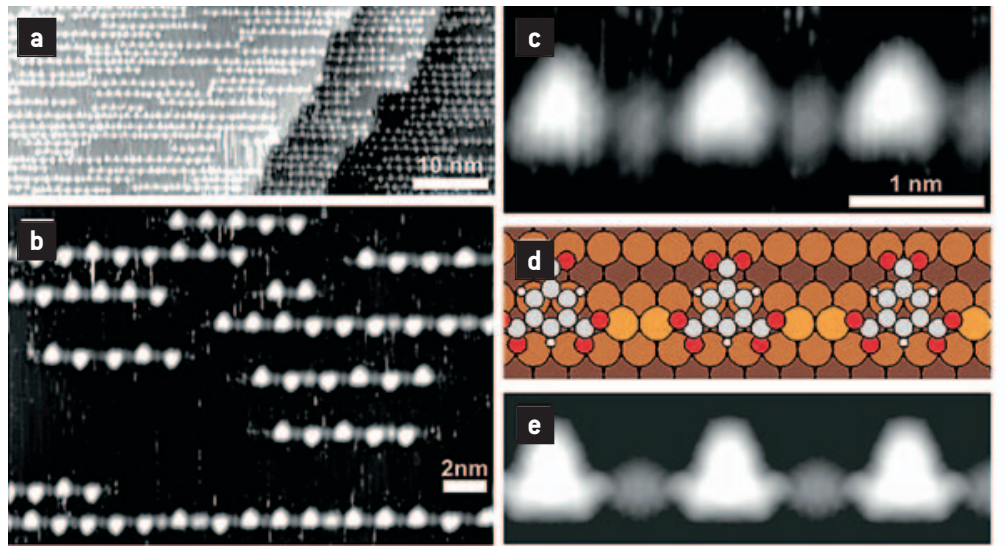

Figure 1. STM images of [-Cu-TMA-Cu-]n chains on $\mathrm{Cu}(110)$ for TMA coverages of [a] 0.36 and [b] 0.13 monolayers $(M L)$, respectively. Comparison of $[\mathbf{c}$ ] the high-resolution STM image, [d] the atomistic model, and e] the corresponding simulated STM image 

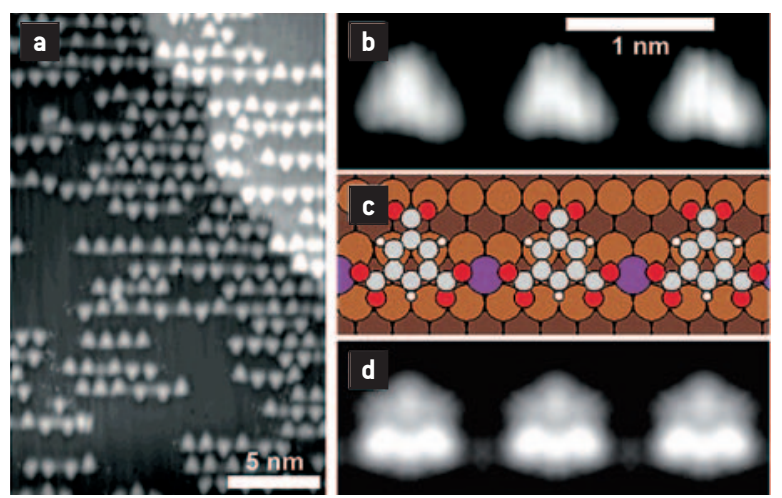

Figure 2. [-TMA-Fe-]n

chains: (a) Overview

image of the

coordination chains

formed upon deposition

of $0.04 \mathrm{MLFe}$ and 0.40

ML TMA. Comparison of

(b) the high-resolution

STM image, [c] the

atomistic model, and [d]

the corresponding

simulated STM image.

Figure 3. (a) Total density of electronic states [DOS, in [eV] with respect to the Fermi energy EF] of the [-TMA-Fe-]n chain. Projected DOS (PDOS) on the atomic $d$ states of (b) the Fe center in MOCC-II and (c) an Fe atom isolated on the $\mathrm{Cu}(110)$ surface.
The chains consist of triangles alternating with round protrusions (Figure 1). The apparent height of the two units is significantly different, $140 \pm 30 \mathrm{pm}$ and $75 \pm 20 \mathrm{pm}$, respectively, when scanning at $1 \mathrm{~V}$ and $1 \mathrm{nA}$. Following previous analysis, the triangles are identified as flat-lying TMA molecules. The round protrusions can be attributed to $\mathrm{Cu}$ adatoms, coordinated by two of the carboxylate groups of the TMA molecule. The third functional group of TMA is pointing out of the chain with no preferential up or down orientation (Figure $2 \mathrm{~b}$ ).

The periodicity of MOCC-I along $<1$ bar10 $>$ is five $\mathrm{Cu}$ lattice spacings $(12.70 \pm 0.15 \AA)$. High-resolution STM images indicate that the distance between the $\mathrm{Cu}$ protrusions and the oxygen atom of the molecular carboxylate groups is approximately $2.8 \AA$, a rather large value when com-

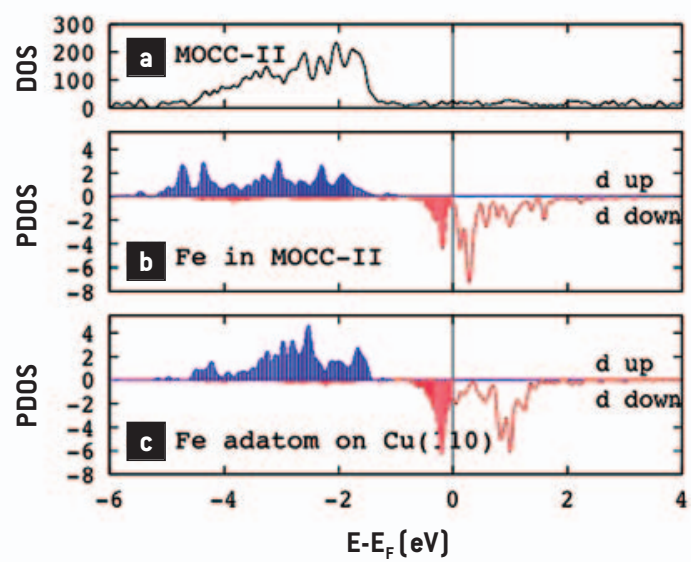

pared to the typical $\mathrm{Cu}-\mathrm{O}$ bond length of 1.9-2.2A. The simplest [-TMA-Cu-]n chain model for the adsorption geometry of MOCC-I seems therefore to be outruled by these observations.

Indeed, the lowest energy structure of MOCC-I as predicted by density functional theory (DFT) calculations is a [-Cu-TMA$\mathrm{Cu}-] \mathrm{n}$ chain in which a dimer of $\mathrm{Cu}$ metal adatoms forms unidentate $\mathrm{Cu}$-carboxylate bonds with adjacent TMA molecules (Figure 1d). The dimer binds to the surface by 6.3 $\mathrm{eV}$ with respect to isolated $\mathrm{Cu}$ atoms, and each adatom is fivefold coordinated to the substrate. This structure has the correct $5 \mathrm{x}$ periodicity. Its simulated STM image (Figure 1e) closely agrees with that found experimentally, with the $\mathrm{Cu}-\mathrm{Cu}$ dimer imaged as a single spot centered between the adatoms. The resulting $\mathrm{Cu}-\mathrm{O}$ distance is $2.02 \AA$, which lies in the range of typical $\mathrm{Cu} \mathrm{O}$ bond lengths. Also the calculated apparent heights, $170 \mathrm{pm}$ for the TMA unit and $90 \mathrm{pm}$ for the $\mathrm{Cu}$ adatom unit, are in good agreement with those determined experimentally.

The theoretical analysis provides an unprecedented level of insight into the adsorption geometry of surface MOCNs. The phenyl ring of TMA and the $\mathrm{Cu}$ adatoms are located on the short-bridge and hollow sites, respectively (Figure 1d). The molecule stands $1.14 \AA$ above the outermost $\mathrm{Cu}$ layer, with the carboxylate groups bending towards the surface by as much as $0.69 \AA$.

[-Cu-TMA-Cu- $\mathrm{n}$ chains are the intrinsic nanostructures on $\mathrm{Cu}(110)$, but functional 
MOCCs also require different elements than $\mathrm{Cu}$ as metallic centers. Extrinsic [TMA-Fe-]n chains (MOCC-II) were created by depositing first TMA and then Fe at coverages higher than $0.04 \mathrm{ML}$.

The $4 \mathrm{x}$ in-chain periodicity of these chains (Figure 2) leaves space for just one $\mathrm{Fe}$ metal center between TMA molecules. According to DFT calculations the geometry of the adsorbed TMA molecule is weakly dependent on the metal center, with the phenyl ring lying 0.09 higher in the case of Fe. The metal-carboxylate bond is still unidentate and the $\mathrm{Fe}-\mathrm{O}$ distance is $1.95 \AA$, thus $0.07 \AA$ shorter than the $\mathrm{Cu}-\mathrm{O}$ distance in MOCC-I. Single metal centers lead to very weak features in the simulated STM image (Figure 2d), in agreement with experiment (Figure 2b).

The projected density of electronic states (DOS) displays a large splitting between the majority spin-up and minority spin-down electronic $d$ states. The former are completely filled and well hybridized with the substrate $\mathrm{Cu} d$ states, extending from 5 to 1 $\mathrm{eV}$ in the total DOS of the [-TMA-Fe-]n chain (Figure 3a). The latter spin-down states are only partially filled and extend in the energy region dominated by the substrate $s$ states. As a consequence, the $\mathrm{Fe}$ atoms are strongly magnetized with a spin polarization of $3.3 \mu_{\mathrm{B}}$ per $\mathrm{Fe}$ atom. The polarization of a Fe adatom isolated on the $\mathrm{Cu}(110)$ surface is very similar, $3.2 \mu_{\mathrm{B}}$. The comparison shows that the coordination with the carboxylate group does not affect the electron localization at the $\mathrm{Fe}$ adatoms and thus does not produce any relevant quenching of the spin magnetic moment.

In conclusion, metal-organic coordination chains were created in situ by selforganized growth at a metal surface under UHV. The $1 \mathrm{D}$ anisotropy of the substrate was effectively transferred to the resulting metal-organic coordination chains. This strategy was shown to work for intrinsic as well as for extrinsic metal-carboxylate systems. The precise atomic configuration of the structures was revealed by a combined use of STM and DFT. Spin-polarized DFT suggests that $\mathrm{Fe}$ centers within the 1D chains have magnetic properties similar to those of isolated $\mathrm{Fe}$ adatoms. This renders such regular and unidirectional arrangement of magnetic centers attractive candidates for the investigation of low-dimensional magnetism in thermally stable structures.

\section{References}

[1] O. M. Yaghi, M. O Eddaoudi, J. Kim, Nature 423, 705 (2003).

[2] A. Dmitriev, H. Spillmann, N. Lin, J. V. Barth, K. Kern, Angew. Chem. 2003, 115, 2774 ; Angew. Chem. Int. Ed. 42, 2670 (2003);

S. Stepanow, M. Lingenfelder, A. Dmitriev, H. Spillmann, E. Delvigne, N. Lin, X. Deng, C. Cai, J. V. Barth, K. Kern, Nat. Mater. 3, 229 (2004).

[3] Th. Classen, G. Fratesi, G. Costantini, S. Fabris, F. L. Stadler, C. Kim, S. de Gironcoli, S. Baroni, K. Kern, Angew. Chem. Int. Ed. 44, 